\title{
Fluorescent-antibody Staining of Conidia of Isolates of Verticillium albo-atrum in Relation to their Virulence for Hop
}

\author{
By SUDESH B. MOHAN* AND JON P. RIDE \\ Department of Microbiology, University of Birmingham, P.O. Box 363, \\ Birmingham B15 2TT, U.K.
}

(Received 6 May 1983; revised 22 July 1983)

\begin{abstract}
Conidial surface antigens from 10 progressive and 10 fluctuating isolates of Verticillium alboatrum were analysed by indirect fluorescence-staining using antisera raised against pooled spores from five isolates of each type. Cross-absorption of antisera with heterologous conidia indicated that the spore surface from eight progressive isolates contained an antigen which was absent from the surface of eight fluctuating isolates. Analysis of single-spore cultures prepared from these isolates by immunofluorescence substantiated the presence of two serological groups of $V$. albo-atrum and indicated that the original cultures were antigenically mixed. The immunofluorescence data could be reconciled with the previously reported immunoelectrophoresis data which indicated the existence of at least three serotypes. There is no correlation, however, between the serotypes and the virulence to hop of V. albo-atrum.
\end{abstract}

\section{INTRODUCTION}

Verticillium albo-atrum (Reinke \& Berthold) infections of hop (Humulus lupulus) may result in either a fluctuating or a progressive type of vascular wilt, attributable to mild (fluctuating) or more virulent (progressive) isolates of the fungus, respectively (Keyworth, 1942; Issac \& Keyworth, 1948). At present the virulence of field isolates can only be assessed by long-term pathogenicity tests (Talboys \& Wilson, 1954; Evans \& Framingham, 1979). In an attempt to develop a more rapid method for distinguishing between progressive and fluctuating isolates, we reported earlier an analysis of mycelial and culture filtrate antigens from the two types of isolate using immunoelectrophoretic techniques (Mohan \& Ride, 1982). On the basis of the precipitin patterns of one antigen, antigen ' 21 ', we described three serotypes for $V$. albo-atrum and correctly predicted the virulence of 16 of the 20 isolates studied.

Sewell \& Wilson (1964) reported that the dispersal of $V$. albo-atrum within the hop plant was due to the transport of conidia in the xylem sap, followed by conidial germination and renewed sporulation. Pelletier \& Hall (1971) reported that spore extracts of $V$. albo-atrum contained at least three proteins which were absent in mycelial extracts. It was possible, therefore, that the presence or absence of conidial surface antigens might be associated with the virulence of progressive isolates. The present study was undertaken to test this possibility and to determine whether any differences detected by fluorescent-antibody staining of the spores could be correlated with the immunoelectrophoretic data reported in the earlier paper.

\section{METHODS}

Organisms and media. The 20 hop isolates of $V$. albo-atrum, 10 progressive ( $P 1$ to $P 10)$ and 10 fluctuating ( $F 1$ to F10), described previously (Mohan \& Ride, 1982), were used in this investigation. Stock cultures were maintained on prune-lactose-yeast extract agar (PLY agar; Talboys, 1960) at $4{ }^{\circ} \mathrm{C}$.

Preparation of conidia. Verticillium albo-atrum isolates were grown on PLY agar slopes for $10 \mathrm{~d}$ at $25^{\circ} \mathrm{C}$ and the conidia collected by flooding the cultures with sterile distilled water, agitating with a loop and filtering the

Abbreviation: RLIE, rocket-line immunoelectrophoresis. 
suspension through four layers of muslin. After centrifuging $(1000 \mathrm{~g}, 5 \mathrm{~min})$ and washing twice, the spores were

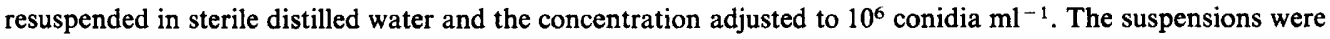
stored at $-20^{\circ} \mathrm{C}$ until required.

Preparation of mycelial antigens. These were prepared as described previously (Mohan \& Ride, 1982).

Production of antisera. Antisera were raised against conidia of the two types of isolate. Pooled spore suspensions $\left(10^{6}\right.$ conidia $\left.\mathrm{ml}^{-1}\right)$ containing equal numbers of conidia from isolates 6 to 10 were used to offset any variation that might occur between isolates of a given type. Three New Zealand white rabbits ( 2 to $3 \mathrm{~kg}$ each) were immunized by intramuscular injection of $1 \mathrm{ml}$ conidial suspension (emulsified with an equal volume of Freund's incomplete adjuvant) at $4 \mathrm{~d}$ intervals for $16 \mathrm{~d}$. A booster injection was given after 4 weeks and the rabbits were bled $10 \mathrm{~d}$ later.

Antisera against pooled mycelial antigens from progressive and fluctuating isolates 6 to 10 were raised as described by Mohan \& Ride (1982).

The immunoglobulin fractions of the antisera were purified by $\left(\mathrm{NH}_{4}\right)_{2} \mathrm{SO}_{4}$ fractionation and chromatography on DEAE-Sephadex-A50 (Pharmacia) according to the method of Harboe \& Ingild (1973). Antisera raised against pooled spores (pooled $P$ spores) and pooled mycelial (pooled PM) antigens from progressive isolates and against the corresponding antigens (pooled $F$ spores and pooled FM) from fluctuating isolates are referred to as anti-P, anti-PM, anti-F and anti-FM, respectively.

Indirect fluorescent-antibody staining. Conidial suspensions $\left(20 \mu \mathrm{l} ; 10^{6}\right.$ spores $\left.\mathrm{ml}^{-1}\right)$ were placed on multitest glass slides (Flow Laboratories), dried at room temperature and then fixed in acetone for $3 \mathrm{~min}$. One drop of antiserum diluted in phosphate-buffered saline (PBS; $0.015 \mathrm{M}$-sodium phosphate, $\mathrm{pH} 7 \cdot 1$, containing $0.15 \mathrm{M}$ $\mathrm{NaCl}$ ) was added to each well and the slides were incubated for $30 \mathrm{~min}$ at room temperature in a moist chamber. The slides were then washed for $30 \mathrm{~min}$ with stirring in two changes of PBS. One drop of fluorescein-conjugated sheep antiserum to rabbit immunoglobulin (Wellcome Reagents) diluted 1:16 in PBS containing, after the initial preliminary experiments, rhodamine counterstain (Difco; diluted $1: 20$ ) was placed on each well and the slides were re-incubated and washed as described above. Finally, the slides were mounted in buffered glycerol ( $0 \cdot 01 \mathrm{M}$-sodium carbonate buffer, $\mathrm{pH} 8$ to 9 , in $90 \%, \mathrm{v} / \mathrm{v}$, glycerol) and examined for fluorescence using a Zeiss fluorescence microscope with excitation filters KP 490 and P 450, and barrier filter LP 520. A positive fluorescence was indicated by a ring of bright green fluorescence around the spores.

Rocket-line immunoelectrophoresis (RLIE). This was as described previously (Mohan \& Ride, 1982).

Cross-absorption of antisera. For cross-absorption with conidia, a spore suspension $(1 \mathrm{ml})$ was centrifuged $(1500 \mathrm{~g}, 5 \mathrm{~min})$, the supernatant decanted, and antiserum $(1 \mathrm{ml})$ mixed with the pellet. After incubation at $37^{\circ} \mathrm{C}$ for $30 \mathrm{~min}$ and then at $4{ }^{\circ} \mathrm{C}$ for $20 \mathrm{~h}$ the absorbed antiserum was centrifuged to remove conidial-antiserum complexes and the supernatant was re-incubated with fresh spores. This procedure was repeated until no fluorescence was obtained in the fluorescent staining test with the absorbed antisera and the spores used for crossabsorption.

Cross absorption with mycelial antigens was as outlined previously (Mohan \& Ride, 1982).

Isolation of single-spore cultures. Conidial suspensions were prepared as described above and diluted in sterile distilled water to give approximately 100 spores $\mathrm{ml}^{-1}$. Aliquots $(0 \cdot 1 \mathrm{ml})$ of the dilute suspension were plated on PLY agar in Petri dishes and incubated at $25^{\circ} \mathrm{C}$. After 2 to $3 \mathrm{~d}$, when some mycelial growth was evident, 10 colonies were picked for each V. albo-atrum isolate, inoculated onto PLY agar slopes and grown for a further week. Agar discs were subcultured on PLY agar slopes for stock cultures which were maintained on this medium and stored at $4{ }^{\circ} \mathrm{C}$. Cultures from each isolate were numbered 1 to 10 ; for example, those from P1 were designated P11, P1-2 etc. to P1-10.

Pathogenicity tests. The pathogenicity of $V$. albo-atrum clones was tested by the Ministry of Agriculture, Fisheries and Food either outdoors as described by Talboys \& Wilson (1954) or in the controlled environment according to the method of Evans \& Framingham (1979). Clones tested by both methods, to provide a cross-check between the two methods used, gave consistent results.

\section{RESULTS}

\section{Fluorescent-antibody staining of pooled progressive or fluctuating isolate spores}

Preliminary experiments using non-immune rabbit serum indicated the necessity of blocking the non-specific reactions of serum with the spore surface. This was accomplished by including the counter stain rhodamine in the fluorescein-conjugated antiserum solution; spores incubated with non-immune serum no longer showed any fluorescence. Pooled spores from both progressive and fluctuating isolates (pooled $\mathrm{P}$ and pooled $\mathrm{F}$ spores, respectively) produced fluorescence when incubated with either anti-P or anti-F at dilutions as high as 1:1024 (Fig. $1 a, c)$. To detect any specific differences between the two types of isolate, the antisera were cross-absorbed with pooled heterologous conidia and then tested for any residual fluorescence 

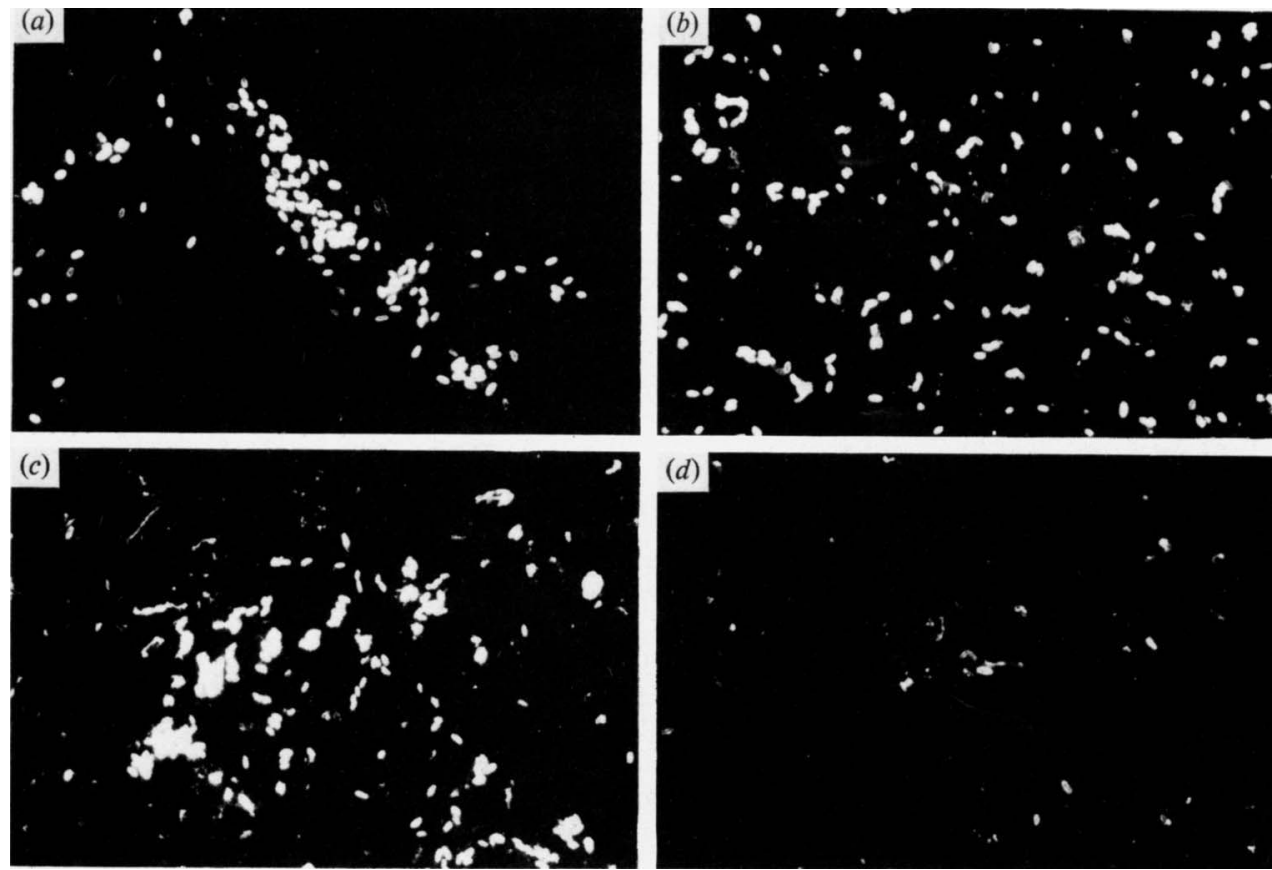

Fig. 1. Immunofluorescence of V. albo-atrum conidia. (a) Pooled $\mathrm{P}$ and (c) pooled F spores stained with anti-P; $(b)$ pooled $\mathrm{P}$ and $(d)$ pooled $\mathrm{F}$ spores stained with anti-P absorbed with pooled $\mathrm{F}$ conidia.

with pooled homologous spores. Cross-absorption of anti-F with pooled $\mathrm{P}$ spores gave an absorbed anti-F which showed faint or no fluorescence with pooled spores from either fluctuating or progressive isolates. In contrast, anti-P absorbed with pooled $F$ spores gave an absorbed anti-P which produced only a faint fluorescence with pooled F spores (Fig. $1 d$ ) but which showed a significant fluorescence with pooled P spores (Fig. 1b). This suggested that there was antigen (or antigens) present on the surface of the conidia of progressive isolates which was absent from the surface of the spores of fluctuating isolates.

\section{Fluorescent-antibody staining of spores from individual isolates}

Conidia from the 20 individual isolates were then assayed in an attempt to determine whether the fluorescence-staining described with absorbed anti-P could be correlated with the virulence of isolates of V. albo-atrum. Absorbed anti-F did not produce any fluorescence with spores from either fluctuating or progressive isolates. With absorbed anti-P (Table 1, column 2), positive fluorescence was obtained with conidia from five fluctuating isolates, F1, F3, F5, F6 and F8, and all progressive isolates except two, P8 and P10. To determine whether non-specific adsorption of serum proteins contributed to these results, antisera were fractionated by $25 \%$ saturated $\left(\mathrm{NH}_{4}\right)_{2} \mathrm{SO}_{4}$. With this partially purified antiserum, the number of positive fluctuating isolates dropped to two, F3 and F6; the two negative progressive isolates, however, remained so (Table 1, column 3). The fluorescence patterns were unchanged following the further purification of the antisera by chromatography on DEAE-Sephadex-A50 (Table 1, column 4). For subsequent experiments, antisera partially purified by $\left(\mathrm{NH}_{4}\right)_{2} \mathrm{SO}_{4}$ fractionation were used.

In an earlier study (Mohan \& Ride, 1982), using RLIE of mycelial antigens from the 20 isolates used in this study in cross-absorbed mycelial antisera, we identified an antigen, antigen ' 21 ', which was present in high concentrations in 12 isolates, absent in four and present in low concentrations in the remaining four isolates. Column 5 in Table 1 compares the present data with those reported earlier. Generally the presence of antigen ' 21 ' correlated with a positive 
Table 1. Fluorescent-antibody staining of spores from individual $V$. albo-atrum isolates using absorbed anti-P

\begin{tabular}{|c|c|c|c|c|}
\hline \multirow[b]{2}{*}{$\begin{array}{l}\text { Isolate } \\
\text { tested }\end{array}$} & \multicolumn{3}{|c|}{ Fluorescence ${ }^{*}$ obtained with } & \multirow[b]{2}{*}{$\begin{array}{c}\text { Antigen ' } 21 \text { ' } \\
\text { patterns in } \\
\text { RLIE† }\end{array}$} \\
\hline & $\begin{array}{c}\text { Whole } \\
\text { antiserum }\end{array}$ & $\begin{array}{l}\text { Antiserum precipitated } \\
\text { with } 25 \% \text { saturated } \\
\left(\mathrm{NH}_{4}\right)_{2} \mathrm{SO}_{4}\end{array}$ & $\begin{array}{l}\text { DEAE-Sephadex-A50 } \\
\text { chromatographed } \\
\text { antiserum }\end{array}$ & \\
\hline P1 & + & + & + & + \\
\hline P2 & + & + & + & + \\
\hline P3 & + & + & + & + \\
\hline P4 & + & + & + & + \\
\hline P5 & + & + & + & + \\
\hline P6 & + & + & + & + \\
\hline P7 & + & + & + & + \\
\hline P8 & - & - & - & - \\
\hline P9 & + & + & + & + \\
\hline P10 & - & - & - & - \\
\hline F1 & + & - & - & - \\
\hline F2 & - & - & - & - \\
\hline F3 & + & + & + & + \\
\hline F4 & - & - & - & + \\
\hline F5 & + & - & - & - \\
\hline F6 & + & + & + & + \\
\hline F7 & - & - & - & $-1+$ \\
\hline F8 & + & - & - & $-1+$ \\
\hline F9 & - & - & - & $-1+$ \\
\hline F10 & - & - & - & $-1+$ \\
\hline
\end{tabular}

* Fluorescence: + , bright-green fluorescence around the spores; - , very dull or no fluorescence around the spores.

† RLIE: +, antigen '21' present in high concentration; - , antigen ' 21 ' absent; $-/+$, antigen ' 21 ' present in low concentration.

fluorescence test, with P10 and F4 being the only anomalies. Furthermore, the virulence of 16 out of 20 isolates could be correctly determined by either test, although both methods falsely identified P8, F3 and F6.

\section{Separate identities of the antigens detected by fluorescence-staining and RLIE}

The high correlation between the fluorescence-staining and RLIE methods suggested either that these methods recognized the same antigen (i.e. antigen ' 21 ') or that two different antigens existed which were perhaps both virulence markers. To investigate these possibilities, antisera raised against mycelial antigens from fluctuating and progressive isolates (pooled FM and pooled PM, respectively; the corresponding antisera being anti-FM and anti-PM) were used. Both anti-FM and anti-PM produced fluorescence with pooled spores from both progressive and fluctuating isolates. Cross-absorption of these antisera with heterologous conidia resulted in absorbed antisera which did not produce fluorescence with either pooled $\mathbf{P}$ or pooled $\mathrm{F}$ spores suggesting that the absorbed antisera did not contain antibodies to any antigens which were specific for the spores from progressive isolates. Nevertheless, the absorbed anti-PM still contained antibody to antigen ' 21 '. This was demonstrated by comparing the precipitin lines formed in RLIE between the spore-absorbed antisera and pooled PM or pooled FM antigens, with the patterns produced by these antigens in anti-PM absorbed with pooled FM (Fig. 2). In the latter antiserum, pooled PM formed a larger rocket (arrowed in Fig. $2 a$ ) than pooled FM. The precipitin pattern of this antigen ' 21 ' could be traced in the patterns formed with anti-PM absorbed with pooled $\mathrm{F}$ spores but not with anti-FM absorbed with pooled $\mathrm{P}$ spores (Fig. $2 b$ and $c$, respectively). The two methods, therefore, detect different antigens and the mycelial antigen ' 21 ' is not responsible for the observed immunofluorescence of progressive spores. 


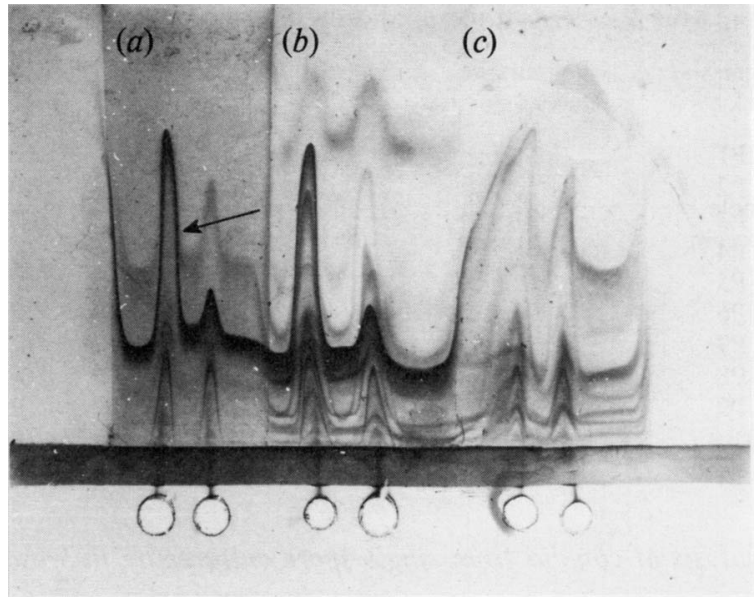

Fig. 2. Comparison by rocket-line immunoelectrophoresis of patterns produced by pooled $V$, alboatrum mycelial antigens against: $(a)$ anti-PM absorbed with pooled FM; $(b)$ anti-PM absorbed with pooled F spores; and $(c)$ anti-FM absorbed with pooled $P$ spores. The wells in each case contained pooled PM (left) and pooled FM (right). The arrow indicates the precipitin line formed by antigen ' 21 '.

\section{Cross-absorption of anti-P and anti- $F$ with spores from isolates $P 8$ and $F 6$}

Attempts were made to explain the false identification of P8, F3 and F6. The first possibility was that these isolates might have been misidentified by the pathogenicity tests. Retesting these isolates proved this possibility to be invalid because the original classification of these isolates was confirmed. Another possibility was that these isolates might represent 'intermediate' groups of $V$. albo-atrum with, for instance, the fluorescence-positive F3 and F6 spores having different surface antigens from the fluorescence-positive progressive group. If this were the case the crossabsorption of anti-P and anti-F with conidia from these isolates should result in absorbed antisera which would not give fluorescence patterns similar to those described above with spores from individual isolates. Cross-absorption of anti-F with spores from either P8 or F6 gave absorbed antisera which showed no fluorescence with spores from any of the 20 isolates. However, anti-P absorbed with spores from P8 resulted in an absorbed antiserum which showed positive fluorescence with spores from F3, F6 and all progressive isolates except P8 and P10, a pattern similar to that obtained with anti-P absorbed with pooled $F$ spores. In contrast, anti-P absorbed with conidia from F6 produced an antiserum which did not produce fluorescence with spores from either progressive or fluctuating isolates. These results suggested that the antigens present on the surface of spores from P8 were similar to those present on fluorescence-negative fluctuating isolates and that the spore surface of F6 (and F3) were antigenically similar to the fluorescence-positive progressive isolatęs. There appeared to be, therefore, two distinct antigenic groups of $V$. albo-atrum as judged by fluorescence-staining.

\section{Percentage spore fluorescence: an indicator of mixed or heterokaryotic cultures?}

The $V$. albo-atrum isolates used in these studies were obtained from infected hop plants simply by the culture of fungus from wedges cut from the vascular tissue. It was possible, therefore, that these cultures might be mixed, that is, they might contain two or more different strains, or they might be heterokaryotic. The observed virulence of the isolates might then be a reflection of the proportion of progressive and fluctuating strains in the mixture. Conidia from individual isolates were therefore analysed for the percentage of spores that showed fluorescence with absorbed anti-P to obtain an indication of the possible extent of mixture (Table 2). With the eight positive progressive isolates the percentage spore fluorescence varied between $53 \%$ and $88 \%$. Only $20 \%$ of spores from P10 produced fluorescence whereas P8 was negative. The two fluctuating isolates which were positive, F3 and F6, had $64 \%$ and $68 \%$ spores fluorescent, respectively, and fell within the range obtained for the progressive isolates. 
Table 2. Percentage of spores showing fluorescence

$\begin{array}{cccc}\begin{array}{c}\text { Progressive } \\ \text { isolates }\end{array} & \begin{array}{c}\text { Percentage } \\ \text { fluorescence }\end{array} & \begin{array}{c}\text { Fluctuating } \\ \text { isolates }\end{array} & \begin{array}{c}\text { Percentage } \\ \text { fluorescence }\end{array} \\ \text { P1 } & 88 & \text { F1 } & 0 \\ \text { P2 } & 53 & \text { F2 } & 0 \\ \text { P3 } & 71 & \text { F3 } & 64 \\ \text { P4 } & 68 & \text { F4 } & 0 \\ \text { P5 } & 54 & \text { F5 } & 0 \\ \text { P6 } & 72 & \text { F6 } & 68 \\ \text { P7 } & 77 & \text { F7 } & 0 \\ \text { P8 } & 0 & \text { F8 } & 0 \\ \text { P9 } & 58 & \text { F9 } & 0 \\ \text { P10 } & 20 & \text { F10 } & 0\end{array}$

Table 3. Analysis of conidia from single-spore cultures by fluorescence-staining

\begin{tabular}{|c|c|c|c|c|c|}
\hline \multirow[b]{2}{*}{ Isolate } & \multicolumn{2}{|c|}{$\begin{array}{l}\text { No. of colonies whose } \\
\text { conidia gave: }\end{array}$} & \multirow[b]{2}{*}{ Isolate } & \multicolumn{2}{|c|}{$\begin{array}{l}\text { No. of colonies whose } \\
\text { conidia gave: }\end{array}$} \\
\hline & $\begin{array}{l}\text { Positive } \\
\text { fluorescence }\end{array}$ & $\begin{array}{l}\text { Negative } \\
\text { fluorescence }\end{array}$ & & $\begin{array}{l}\text { Positive } \\
\text { fluorescence }\end{array}$ & $\begin{array}{l}\text { Negative } \\
\text { fluorescence }\end{array}$ \\
\hline P1 & 9 & 1 & F1 & 0 & 10 \\
\hline P2 & 6 & 4 & F2 & 0 & 10 \\
\hline P3 & 10 & 0 & F3 & 10 & 0 \\
\hline P4 & 7 & 3 & F4 & 0 & 10 \\
\hline P5 & 8 & 2 & F5 & 0 & 10 \\
\hline P6 & 9 & 1 & F6 & 6 & 4 \\
\hline P7 & 10 & 0 & F7 & 0 & 10 \\
\hline P8 & 0 & 10 & F8 & 0 & 10 \\
\hline P9 & 10 & 0 & F9 & 0 & 10 \\
\hline P10 & 4 & 6 & F10 & 0 & 10 \\
\hline
\end{tabular}

Analysis of single-spore cultures by fluorescence-staining and RLIE

If the data shown in Table 2 are a true indication of the degree of mixture or heterokaryosis of the isolates, then, since V. albo-atrum conidia are known to be haploid uninucleate cells (Roth \& Brandt, 1964; Buckley et al., 1969), it should be possible to isolate single-spore cultures which show either a $100 \%$ or $0 \%$ fluorescence of conidia when tested with absorbed anti-P. To determine whether this was the case, 10 single-spore cultures were prepared from each of the 20 isolates and the conidia from these tested for fluorescence (Table 3). Six out of the 10 progressive isolates (P1, P2, P4, P5, P6 and P10) showed evidence of being mixed or heterokaryotic. The proportion of single-spore isolates with fluorescence-negative spores varied from one out of 10 in $\mathrm{P} 1$ and $\mathrm{P} 6$ to six out of 10 in $\mathrm{P} 10$. The remaining four progressive isolates (P3, P7, P8 and P9) appeared to be pure: spores from all P8 colonies were fluorescence-negative in contrast to those from the remaining three isolates which were positive. With the positive colonies, $100 \%$ of the spores showed fluorescence and this remained so on subsequent subculturing. Single-spore cultures from eight fluctuating isolates produced fluorescence-negative conidia. Of the remaining two, F3 produced all positive colonies whereas $\mathrm{F} 6$ was mixed.

Mycelial antigens from some of the single-spore cultures were next tested by RLIE to determine whether the high correlation observed earlier between the two methods was again apparent. Four out of the 10 P10 clones tested (P10-1, P10-4, P10-5 and P10-9) formed rockets corresponding to antigen ' 21 ' (shown by an arrow in Fig. $3 a$ ). This rocket was barely detected in P10-2 and was absent in the remaining five cultures. Antigen ' 21 ' was also present in large concentrations in six out of 10 F6 cultures (F6-1, F6-2, F6-7, F6-8, F6-9 and F6-10; Fig. 3b) and in all of the seven P6 cultures tested except P6-1 which formed a smaller rocket, suggesting that 

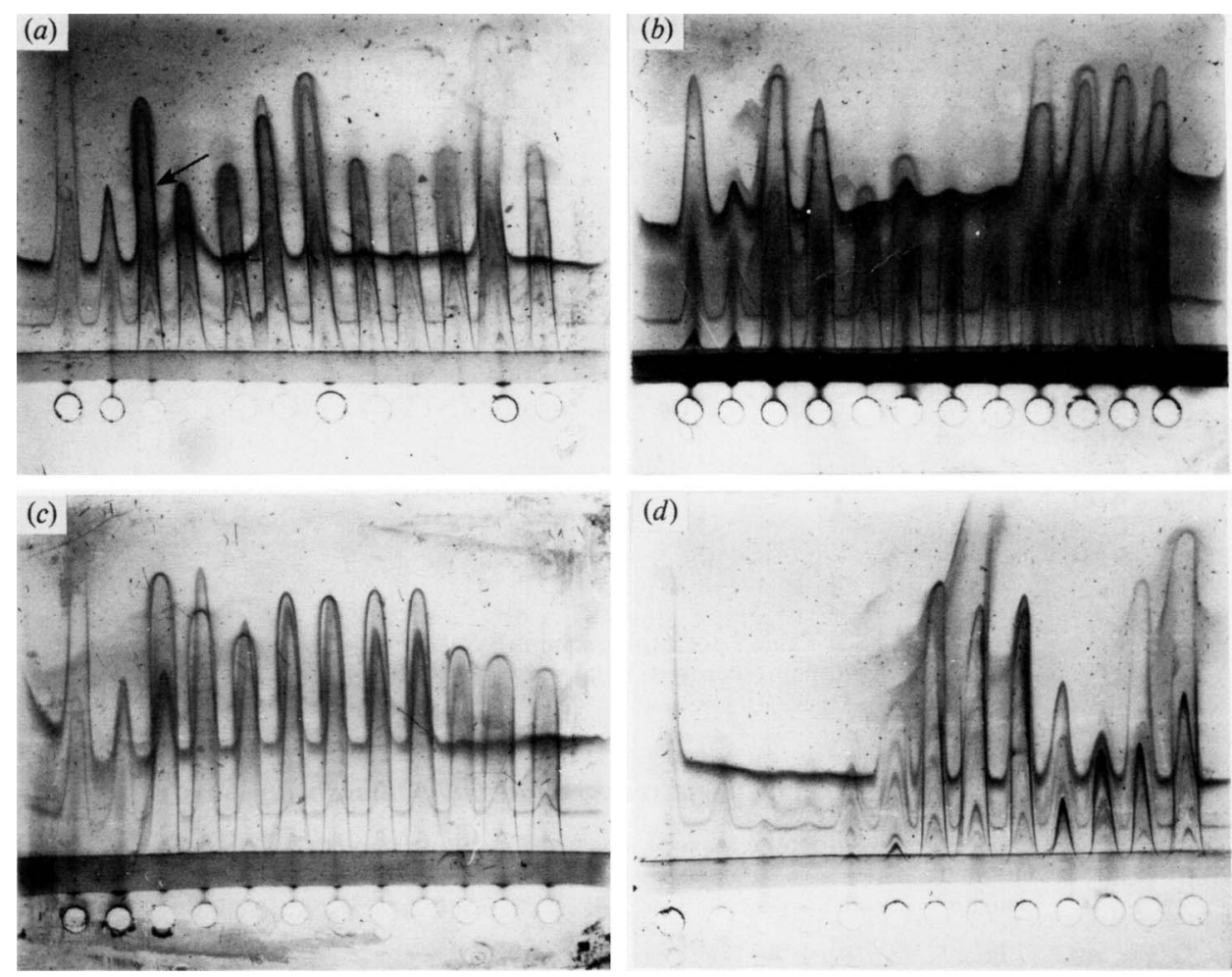

Fig. 3. Rocket line immunoelectrophoresis of mycelial antigens from single-spore cultures run against anti-PM absorbed with pooled FM with pooled PM in the intermediate gel. The wells contained (from left to right): (a) pooled PM, pooled FM and mycelial extracts from P10-1 to P10-10; $(b)$ pooled PM, pooled FM and mycelial extracts from F6-1 to F6-10; (c) pooled PM, mycelial extracts from P6-1 to P6-7 and F9-4 to F9-6; and (d) pooled PM, and mycelial extracts from F7-1, F7-5 to F7-7, F8-1, F8-5, F8-6, F8-10, F10-1, F10-5, F10-7 and F10-8. The arrow indicates the precipitin line formed by antigen ' 21 '.

less antigen was present in this clone (Fig. $3 c$ ). Antigen ' 21 ' was not detected in the four F7 (F71, F7-5, F7-6 and F7-7) and in the three F9 (F9-4, F9-5 and F9-6) colonies tested (Fig. $3 d$ and $c$, respectively). Extracts from four clones each of $F 8$ and $F 10$ isolates contained varying concentrations of antigen '21' (Fig. 3d). Three F8 clones (F8-5, F8-6 and F8-10) and one F10 culture (F10-7) contained large concentrations of antigen ' 21 '. In contrast, in F8-1, F10-1, F10-5 and F10-8, low concentration of antigen ' 21 ' was present.

Table 4 compares the fluorescence patterns obtained for the single-spore cultures tested in RLIE. All clones showing positive spore fluorescence contained high concentrations of antigen ' 21 ' thus substantiating the earlier observations (Table 1). Negative fluorescence was accompanied by either the absence or presence of antigen ' 21 '.

\section{Identification of single-spore cultures by pathogenicity tests}

Nineteen clones were tested by the pathogenicity tests (Table 5) to determine whether the correlation observed between the fluorescence staining and RLIE detected antigens could be associated with the virulence of these cultures. The tests identified the two P6 and the six P10 clones tested as progressive and the six F6, one F9 and two clones each of F8 and F10 as fluctuating. These results suggested that the distinct antigenic groups observed did not correspond to the progressive/fluctuating distinction made on the basis of virulence. 
Table 4. Comparison of fluorescence and RLIE patterns obtained for single-spore cultures

\begin{tabular}{|c|c|c|c|c|c|c|c|c|c|c|c|}
\hline \multirow{2}{*}{$\begin{array}{l}\text { Original } \\
\text { isolate }\end{array}$} & \multirow[b]{2}{*}{ Test } & \multicolumn{10}{|c|}{ Patterns obtained with single-spore culture number:* } \\
\hline & & 1 & 2 & 3 & 4 & 5 & 6 & 7 & 8 & 9 & 10 \\
\hline \multirow[t]{2}{*}{ P6 } & RLIE & $-1+$ & + & + & + & + & + & + & & & \\
\hline & FS $\dagger$ & - & + & + & + & + & + & + & & & \\
\hline \multirow[t]{2}{*}{ P10 } & RLIE & + & $-1+$ & - & + & + & - & - & - & + & - \\
\hline & FS & + & - & - & + & + & - & - & - & + & - \\
\hline \multirow[t]{2}{*}{ F6 } & RLIE & + & + & - & - & - & - & + & + & + & + \\
\hline & FS & + & + & - & - & - & - & + & + & + & + \\
\hline \multirow[t]{2}{*}{ F7 } & RLIE & - & & & & - & - & - & & & \\
\hline & FS & - & & & & - & - & - & & & \\
\hline \multirow[t]{2}{*}{ F8 } & RLIE & $-1+$ & & & & + & + & & & & + \\
\hline & FS & - & & & & - & - & & & & - \\
\hline \multirow[t]{2}{*}{ F9 } & RLIE & & & & - & - & - & & & & \\
\hline & FS & & & & - & - & - & & & & \\
\hline \multirow[t]{2}{*}{ F10 } & RLIE & $-1+$ & & & & $-1+$ & & + & $-1+$ & & \\
\hline & FS & - & & & & - & & - & - & & \\
\hline
\end{tabular}

* See Table 1 for explanation of symbols.

† FS, fluorescence-staining.

Table 5. Identification of single-spore cultures by pathogenicity tests

\begin{tabular}{|c|c|c|c|c|c|c|c|c|}
\hline \multirow{2}{*}{$\begin{array}{c}\text { Original } \\
\text { isolate }\end{array}$} & \multicolumn{8}{|c|}{ Identification of single-spore culture number:* } \\
\hline & 1 & 2 & 3 & 4 & 5 & 6 & 7 & 10 \\
\hline P6 & $\mathbf{P}$ & & & $\mathbf{P}$ & & & & \\
\hline P10 & $\mathbf{P}$ & $\mathbf{P}$ & $\mathbf{P}$ & $\mathbf{P}$ & $\mathbf{P}$ & $\mathbf{P}$ & & \\
\hline F6 & $\mathrm{F}$ & $\mathbf{F}$ & $\mathbf{F}$ & $\mathbf{F}$ & $\mathrm{F}$ & $\mathbf{F}$ & & \\
\hline F8 & & & & & $\mathrm{F}$ & & & $\mathbf{F}$ \\
\hline F9 & & & & & $\mathbf{F}$ & & & \\
\hline F10 & & & & & $\mathrm{F}$ & & $\mathbf{F}$ & \\
\hline
\end{tabular}

\section{DISCUSSION}

The indirect fluorescent-antibody staining method was used successfully in this study to demonstrate antigenic differences on the spore surfaces of the $V$. albo-atrum isolates studied. Anti-P absorbed with pooled $F$ spores produced fluorescence with eight of the 10 progressive isolates (Table 1). In contrast, eight fluctuating isolates were negative. On the basis of fluorescence therefore, the virulence of 16 out of 20 isolates could be identified correctly. Comparison of these data with those reported above by RLIE (Table 1, column 5), in which nine progressive and seven fluctuating isolates were identified correctly as compared with eight of each by fluorescence-staining, shows a $90 \%$ correlation between the two methods.

The antigens detected by the two methods were shown to be different both by crossabsorption tests and by the isolation of single-spore cultures which differed in their antigenic content. The fluorescence-staining data can be used to characterize further the three serological groups described earlier (Mohan \& Ride, 1982). Group 1 was formed by 12 isolates (nine progressive and three fluctuating) which contained large concentrations of antigen ' 21 ', (Table 1). All except two (P10 and F4) of these were also positive by fluorescence-staining: serotype 1 is now, therefore, both RLIE and fluorescence positive. Group 2 was formed by five isolates which were negative for antigen ' 21 ' (P8, F1, F2, F5 and F8); these were also negative by fluorescence. Hence this serotype is negative by both methods. Serotype 3 contained four isolates (F7, F8, F9 and F10) which had low concentrations of antigen '21'; these were also fluorescence-negative. The two anomalies, P10 and F4, were both positive by RLIE but negative by fluorescence-staining. These can be incorporated into serotype 3 if this group is 
described as RLIE-positive and fluorescence-negative. The analysis of single-spore cultures by the two methods (Table 4) provided further evidence that these three serotypes are characteristic of $V$. albo-atrum isolates from hop.

Sixteen out of the 20 original isolates were identified correctly using the three serotypes. It was interesting that both methods used detected $P 8$ as a false negative progressive and F3 and $F 6$ as false positive fluctuating isolates. We had suggested earlier that these isolates could be either misidentified by pathogenicity tests or that they could represent mixed cultures. Repeating the pathogenicity tests did not change the original virulence assignments, but the examination of single-spore cultures did indicate that the original isolates were mixed or heterokaryotic, at least as far as their antigenic properties were concerned. Tests on the virulence of single-spore cultures from different serotypes, however, showed no correlation between the antigen content and virulence of the cultures, indicating that the original results which appeared to be $80 \%$ predictive were purely fortuitous. In fact, progressive and fluctuating strains can apparently belong to any of the three immunotypes. These three immunotypes probably reflect serotypes similar to those found, for example, in streptococci where antigenic variations of immunotypes are not correlated with pathogenicity (Lancefield, 1933). It is probable, therefore, that successful serological differentiation of fluctuating and progressive isolates might depend on the selection of appropriate antigens. Enzymes and other macromolecules produced by the fungus when growing in the host may prove to be the most relevent antigens for these tests. Isolates from the three immunotypes are being characterized further at present, in an attempt to exploit this possibility.

The authors are grateful to Dr D. L. Ebbels, Ministry of Agriculture, Fisheries and Food, Harpenden Laboratory, Hatching Green, Harpenden, U.K., for pathogenicity tests, and to the Ministry of Agriculture, Fisheries and Food for financial support.

\section{REFERENCES}

Buckley, P. M., Wyllie, T. D. \& Devay, J. E. (1969). Fine structure of conidia and conidium formation in Verticillium albo-atrum and $V$. nigrescens. Mycologia 61, 240-250.

Evans, E. J. \& Framingham, E. E. (1979). Method of determining strain pathogenicity of Verticillium alboatrum wilt of hops. Technical Bulletin, Ministry of Agriculture, Fisheries and Food, South Eastern Region.

HARBOE, N. \& INGILD, A. (1973). Immunization, isolation of immunoglobulins, estimation of antibody titre. Scandinavian Journal of Immunology 2, suppl. 1, 161-164.

ISSAC, I. \& KEYWORTH, W. G. (1948). Verticillium wilt of hop (Humulus lupulus). III. A study of the pathogenicity of isolates from fluctuating and from progressive outbreaks. Annals of Applied Biology 35, 243-249.

KEYWORTH, W. G. (1942). Verticillium wilt of the hop (Humulus lupulus). Annals of Applied Biology 29, 346357.

LANCEFIELD, R. C. (1933). A serological differentiation of human and other groups of hemolytic streptococci. Journal of Experimental Medicine 57, 571-595.
MoHan, S. B. \& Ride, J. P. (1982). An immunoelectrophoretic approach to the identification of progressive and fluctuating isolates of the hop wilt fungus Verticillium albo-atrum. Journal of General Microbiology 128, 255-265.

Pelletier, G. \& Hall, R. (1971). Relationships among species of Verticillium: protein composition of spores and mycelium. Canadian Journal of Botany 49, 1293-1297.

ROTH, J. N. \& BRANDT, W. H. (1964). Nuclei in spores and mycelium of Verticillium. Phytopathology 54, 363-364.

Sewell, G. W. F. \& Wilson, J. F. (1964). The occurrence and dispersal of Verticillium conidia in xylem sap of the hop (Humulus lupulus). Nature, London 204, 901.

TALBOYS, P. W. (1960). A culture medium aiding the identification of Verticillium albo-atrum and $V$. dahliae. Plant Pathology 9, 57-58.

TAlboYS, P. W. \& WILSON, J. F. (1954). A method for determining the pathogenicity of strains of Verticillium albo-atrum isolates from hop. Report East Malling Research Station for 1953, 158-161. 